\title{
Should I Change or Should I Go? Phenotypic Plasticity and Matching Habitat Choice in the Adaptation to Environmental Heterogeneity
}

\author{
Pim Edelaar, ${ }^{1,2, \star, \dagger}$ Roger Jovani, ${ }^{2, \star}$ and Ivan Gomez-Mestre ${ }^{2, \dagger}$ \\ 1. University Pablo de Olavide, Seville, Spain; 2. Estación Biológica de Doñana, Consejo Superior de Investigaciones Científicas, \\ Seville, Spain \\ Submitted October 22, 2016; Accepted April 14, 2017; Electronically published August 17, 2017 \\ Online enhancements: appendix. Dryad data: http://dx.doi.org/10.5061/dryad.g370c.
}

\begin{abstract}
It can be challenging for organisms to achieve a good match between their phenotypic characteristics and environmental requirements that vary in space and time. The evolution of adaptive phenotypes can result from genetic differentiation at the population level. Individuals, however, could also change their phenotype (adaptive plasticity) or select an environment because it matches with their phenotype (matching habitat choice). It is poorly known under which conditions these different solutions to environmental heterogeneity evolve and whether they operate together. Using an individual-based simulation model, we assessed which solutions evolved depending on degree of temporal variation, costs of multiple underlying traits, and order of dispersal and development. Population genetic divergence was superseded by plasticity or matching habitat choice as temporal variation increased. Plasticity and matching habitat choice were limited by their trait costs, even when this involved only a part of the underlying traits. Independent of the order of dispersal and development, plasticity evolved more commonly than matching habitat choice, in part because the match a phenotype can achieve by matching habitat choice is limited by the types of environments available. Our results explain the apparent relative rarity of matching habitat choice in nature. At the same time, our results can be used to look for matching habitat choice in those biological systems where the conditions for other solutions seem unfavorable.
\end{abstract}

Keywords: phenotypic plasticity, matching habitat choice, environmental heterogeneity, dispersal, local adaptation, individual-based simulation model.

The degree to which organisms are adapted to the environmental conditions that they encounter affects their ecological interactions and their fitness (Rose and Lauder 1996).

\footnotetext{
* These authors contributed equally.

† Corresponding authors; e-mail: Edelaar, edelaar@upo.es; Gomez-Mestre, igmestre @ebd.csic.es.

ORCIDs: Edelaar, http://orcid.org/0000-0003-4649-6366; Gomez-Mestre, http:// orcid.org/0000-0003-0094-8195.
}

Am. Nat. 2017. Vol. 190, pp. 506-520. (C) 2017 by The University of Chicago. 0003-0147/2017/19004-57344\$15.00. All rights reserved.

DOI: $10.1086 / 693345$
How individuals and populations maximize their local performance given environmental heterogeneity in time and space is therefore of great interest in ecology and evolution. Local performance depends on the interaction between phenotypes and environments. Therefore, there are several routes toward enhanced local performance, depending on whether it is the phenotypes that are being adjusted or whether it is the environments that are being selected or modified. With respect to phenotypic adjustment, natural selection can favor some phenotypes over others, and if there is genetic variation underlying phenotypic variation, such selection will result in locally adapted, genetically differentiated populations (Kawecki and Ebert 2004; Räsänen and Hendry 2008).

However, several mechanisms may evolve that allow individuals, and not just populations, to increase their local performance when faced with environmental variation. Thus, organisms may alter gene expression in response to environmental stimuli, adaptively modifying their behavior, morphology, physiology, and/or life histories to match local conditions. Such adaptive plasticity (hereafter, plasticity) readily evolves under environmental heterogeneity (Thibert-Plante and Hendry 2010; Gomez-Mestre and Jovani 2013; Hendry 2015). Nevertheless, the evolution of plasticity requires reliable cues to assess environmental conditions, a heritable basis of plastic responses, and that the benefits of being plastic outweigh the costs of plasticity (Pigliucci 2001; West-Eberhard 2003). Plasticity has been well documented across many taxa and might be key in the origin of evolutionary innovations and subsequent diversification and speciation through genetic accommodation (West-Eberhard 2003; Gomez-Mestre and Buchholz 2006; Moczek et al. 2011).

Local performance can also be enhanced by adaptively adjusting (including choosing) the environment in which an individual will perform. Habitat choice is a well-documented, highly relevant attribute of species and locally adapted populations (e.g., Morris 2003; Morin 2011). Moreover, when individuals differ in phenotype, their habitat of choice may 
also differ. Phenotype- and context-dependent habitat choice that results from self-assessment and comparison of performance across habitats and that increases individual performance has been termed matching habitat choice (MHC; Ravigné et al. 2004; see Edelaar et al. 2008 for an overview of alternative terms). In contrast to other forms of habitat choice (e.g., due to imprinting or fixed genetic preferences), MHC depends on phenotypic differences among individuals and some sort of assessment of local performance. As is true for plasticity, such adaptive MHC should be able to evolve in response to environmental heterogeneity, but MHC evolution requires reliable cues to assess local performance, a heritable basis of the sensory and behavioral responses to local performance variability, and that the benefits of choosing a more adequate habitat outweigh any potential costs of choice, including movement.

MHC and plasticity could be seen as alternative and orthogonal solutions to increase local performance (Edelaar et al. 2008): plasticity changes an individual's phenotype to match its environment, whereas MHC changes an individual's environment to match its phenotype. Local adaptation due to natural selection on nonplastic, nonchoosing genotypes is then the null situation: individuals neither change their phenotype nor choose their environment, and therefore a good phenotype-environment match is not actively achieved by individuals but passively attained by populations through differential reproduction of genetic variants with different phenotypes. Plasticity and MHC may evolve and exert their influence on top of this background process of adaptation by genetic differentiation due to natural selection, especially when environmental heterogeneity is high and populations are arrayed in metapopulations (Sultan and Spencer 2002; Ravigné et al. 2009; Gomez-Mestre and Jovani 2013).

Plasticity and MHC have been studied empirically and theoretically (e.g., Pigliucci 2001; Sultan and Spencer 2002; West-Eberhard 2003; Edelaar et al. 2008; Ravigné et al. 2009; Karpestam et al. 2012; Scheiner et al. 2012; Bolnick and Otto 2013), yet not together, except for Scheiner $(2013,2016)$ and this study. Such a synthesis is clearly needed, since both mechanisms try to solve the same challenge in very different ways. In general, not allowing for the simultaneous evolution of multiple solutions to single problems can severely bias our perception of the relevance of each solution (Rueffler et al. 2006). Intuitively, one could expect the simultaneous evolution of both strategies, because they might increase individual fitness as each mechanism improves the imperfections of the other. Alternatively, if there are costs associated to these mechanisms, one might hypothesize that organisms may not evolve a mechanism that they cannot use, do not need, or which might even interfere with the correct operation of the other mechanism. To improve our understanding of the evolution of MHC and plasticity alike, and to stimulate the mod- eling of the simultaneous evolution of multiple solutions to single problems, we therefore investigate here, in a single model, whether and how MHC and plasticity evolve when genetic local adaptation through natural selection is also a potential solution. In this model, we varied the order in which plasticity and MHC operated, the degree of temporal environmental variation, and the costs of the traits underlying plasticity and $\mathrm{MHC}$ to test their effects on adaptive evolution. However, since the order of occurrence of developmental plasticity and MHC did not qualitatively affect the results, and because MHC is unlikely to occur in nature before developmental plasticity, we do not further discuss this aspect in much detail below and refer to figure A1 (available online) for the comparison. To avoid favoring the evolution of plasticity or MHC over one another, we modeled the evolution of both strategies, making their underlying traits as comparable as possible in their operation, in their initial and potential variability, and in their costs. In these respects, our study differs importantly from the recent modeling approach by Scheiner (2016). Additionally, our study differs in that habitat choosiness and the degree of dispersal are unlinked, individuals move directionally toward a better habitat or phenotype, and the capacity to assess the degree of mismatch can evolve.

\section{Material and Methods}

\section{Model Implementation}

We implement an individual-based simulation modeling approach. An advantage of this approach over analytical models (besides the possibility of incorporating many more realistic details) is that many aspects are stochastic rather than fixed proportions, so the effects of the full distributions of individual fitness can be taken into account (Starrfelt and Kokko 2012). Our model is an abstraction of reality that includes those features that we considered to be important a priori, allowing us to capture and understand general patterns. We model a metapopulation whose patch environments can vary in time, inhabited by organisms with a phenotypic trait that affects ecological performance. These organisms can evolve dispersal, plasticity, and MHC to deal with environmental variability. However, the traits underlying dispersal, plasticity, and MHC can have costs. The relative fit between environment and phenotype, penalized for any trait costs, affects reproductive success. The source code and executable file of the model used here are available also as supplementary material (deposited in the Dryad Digital Repository, http://dx.doi.org/10.5061/dryad.g370c; Edelaar et al. 2017). The model is implemented in NetLogo 5.0.5 (Wilensky 1999). NetLogo is freely downloadable from http://ccl.northwestern.edu/netlogo/download.shtml. Our open-source model, when opened in NetLogo, has a graphical interface that allows users to easily change the settings 
of several parameters (even during a simulation run) and shows, in real time, summary graphs of several output variables. Downloading our model therefore allows corroboration of our conclusions, further investigation, or use in outreach or teaching.

\section{Environment}

Simulations start with a population of 10,000 individuals randomly distributed among 100 habitat patches, each receiving 100 individuals (see fig. 1). Environmental conditions of each patch are indicated by the value of the continuous variable environment (see table 1 for an overview of important model variables). The environment is modeled as a vector with a range of $0^{\circ}-360^{\circ}$. Although there are perhaps no clear empirical equivalents to such a construct (although color and periodicity could be viewed as circular traits), it provides us with a flat distribution for environmental condi-
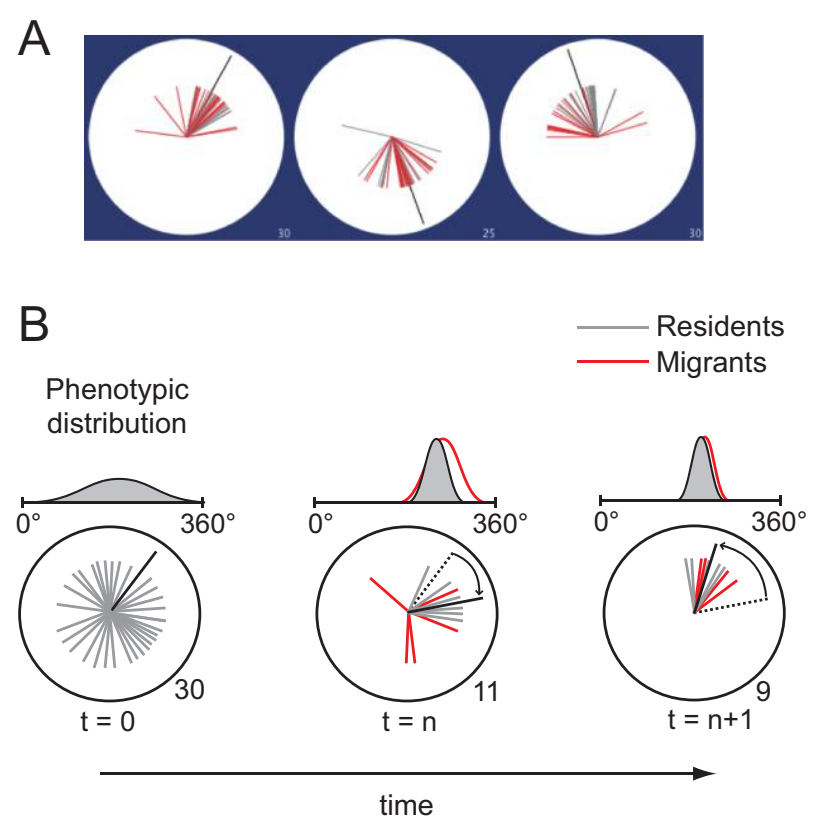

Figure 1: $A$, Snapshot of the visual output of our model. Within each patch (white pies, with no. individuals indicated), the long black line indicates the local environmental state $\left(0^{\circ}-360^{\circ}\right)$, short gray lines indicate the phenotype of nondispersing individuals, and short red lines do so for immigrants. Here, most populations are already quite well matched to their local environment. $B$, Example of events occurring over a simulation at any given patch. At initialization $(t=0)$, a patch receives a random environmental value and 100 individuals, each with a random genotype $\left(0^{\circ}-360^{\circ}\right)$. Each generation, the environment shifts according to the environmental stochasticity set, as shown at $t=1$. According to their evolving characteristics, individuals can exhibit differential reproduction and/or plasticity in phenotype within patches or matching habitat choice between patches. This may result in local populations tracking the environment (as in $t=2$ ), also indicated by the shift in phenotypic distribution. tions that avoids the evolution of a specialist genotype utilizing the most common environment. It further avoids problematic boundary effects during environmental change and helps to maintain the same variability across patches over time. Modeling a circular one-dimensional environment is a common approach in physics (U. Dieckmann, personal communication) and resembles the common modeling of a donut-shaped torus in two-dimensional spatially explicit models.

Patches start with a random environment between $0^{\circ}$ and $360^{\circ}$ (fig. 1). Each generation, patches change their environment according to the value of a random draw from a normal distribution with mean $=0$ and standard deviation $=$ temporal-heterogeneity. A small standard deviation results in temporal autocorrelation per patch and therefore a certain predictability of the new environment for individuals living there, whereas a high one removes autocorrelation and predictability. These temporal changes are unrestricted across the $0^{\circ} / 360^{\circ}$ boundary (i.e., a change from $3^{\circ}$ to $357^{\circ}$ is just a $6^{\circ}$ change). To further avoid certain boundary effects in the evolution of dispersal, we model dispersal capacity not as the distance that an individual can move but as the number of patches it explores (see below). Therefore, there is no spatial structure to the patches; that is, there are no variable interpatch distances, just as in Wright's (1931) classical island model in population genetics.

\section{Individual Characteristics}

Individuals are asexual and haploid, and each inherits five unlinked traits from its parent: a genotype for a functional (ecological) trait; plasticity-potential and plasticity-habitatsensitivity, which together shape developmental plasticity; and dispersal-potential and dispersal-habitat-sensitivity, which together shape MHC. Modeling MHC and plasticity as a two-component process whose elements may evolve independently allows for the different components of the dispersal and plasticity process to have different costs (see below). Genotype values follow a circular distribution (range: $0^{\circ}-360^{\circ}$ ), allowing computationally easy tracking and matching with the environment values in each patch (fig. 1). The other four traits range from 0 (not functional) to 1 (maximally functional). These five traits mutate, and mutation is modeled by extracting a pseudorandom number from a negative exponential distribution (Eyre-Walker and Keightley 2007) characterized by the parameter mean-mutationalchange (table 1). For a negative exponential, small values are much more common than large values: to some extent, this mimics the effects of a lower mutation rate for each of the many loci underlying a quantitative trait and the increased quantitative genetic variation in offspring generated by recombination in sexual organisms. For genotype, the mutational effect is randomly assigned a positive or negative value 
Table 1: Variables and parameterization used for simulations

\begin{tabular}{|c|c|c|}
\hline Variable & Range values & Description \\
\hline \multicolumn{3}{|l|}{ Parameter: } \\
\hline Temporal-heterogeneity & {$[0,400]$} & $\begin{array}{l}\text { Determines the degree of temporal environmental change; } \text { environment }_{t+1}= \\
\text { environment } t_{t}+\mathrm{N} \sim(0 \text {, temporal-heterogeneity }) .\end{array}$ \\
\hline Costs & {$[0,0.1]$} & $\begin{array}{l}\text { The costs associated with (and multiplied by) plasticity-potential, plasticity- } \\
\text { habitat-sensitivity, dispersal-potential, and plasticity-habitat-sensitivity. }\end{array}$ \\
\hline Mean-mutational-change & 0.01 & $\begin{array}{l}\text { Mutation is modeled by extracting a pseudorandom number from an exponential } \\
\text { decay distribution with mean mean-mutational-change and adding it to or } \\
\text { subtracting it from each inherited trait with equal probability. }\end{array}$ \\
\hline \multicolumn{3}{|l|}{ Dynamic variables: } \\
\hline Environment & {$\left[0^{\circ}, 360^{\circ}\right]$} & Single variable describing the features of the habitat patch. \\
\hline Phenotype & {$\left[0^{\circ}, 360^{\circ}\right]$} & Individual trait; without plasticity, phenotype = genotype. \\
\hline Mismatch & {$[0,1]$} & $\begin{array}{l}\text { Mismatch }=\text { absolute value of (environment - phenotype) } / 180(\text { since the maxi- } \\
\left.\text { mum distance between environment and phenotype is } 180^{\circ}\right) \text {. }\end{array}$ \\
\hline \multicolumn{3}{|l|}{ Individual heritable traits: } \\
\hline Genotype & {$\left[0^{\circ}, 360^{\circ}\right]$} & Affects the phenotype to utilize the environment. \\
\hline Plasticity-potential & {$[0,1]$} & Proportion by which maximum mismatch $\left(180^{\circ}\right)$ can be eliminated by plasticity. \\
\hline Plasticity-habitat-sensitivity & {$[0,1]$} & $\begin{array}{l}\text { Proportion of possible values of the environment that is excluded from the as- } \\
\text { sessment of the patch in which an individual develops, starting with the value } \\
\text { that is most incorrect. }\end{array}$ \\
\hline Dispersal-potential & {$[0,1]$} & $\begin{array}{l}\text { Probability that a given nonnatal patch is included in the set of patches to which an } \\
\text { individual could potentially disperse. }\end{array}$ \\
\hline Dispersal-habitat-sensitivity & {$[0,1]$} & $\begin{array}{l}\text { Proportion of possible values of the environment that is excluded from the as- } \\
\text { sessment of each of the patches to which an individual could potentially dis- } \\
\text { perse, per patch, starting with the value that is most incorrect. }\end{array}$ \\
\hline
\end{tabular}

and multiplied by 180 (the maximum change possible for a circular trait). Therefore, it can mutate, for example from $5^{\circ}$ to $358^{\circ}$, or reverse. For the other four traits, the mutational effect is also randomly assigned a positive or negative value but is multiplied by 1 (the maximum change possible on the scale from 0 to 1 ); if the expected new mutant values are $<0$ or $>1$, they are rescaled to receive a new value of 0 or 1 , respectively. At the beginning of a simulation run, each individual receives a random draw from the range $0^{\circ}-360^{\circ}$ for its genotype and a random draw from the range $0-1$ for the other traits. It is subsequently placed in a random patch. Hence all genetic trait values and the environment are completely uncorrelated at the start of a simulation run (fig. 1B). Also, genetic variation is maximized within patches, which allows for a faster evaluation of the final evolutionary outcome (as we do not focus on evolutionary dynamics here).

\section{Plasticity and Dispersal}

Figure 2 shows a schematic diagram of the sequence of events across one generation. In brief, individuals develop first and then disperse. Development translates the genotype into phenotype, and here plasticity may improve the match between the phenotype and the environment (fig. 3). For this, individuals use their plasticity-habitat-sensitivity to exclude a range of environmental values that are certainly incorrect, and subsequently guessing a random environmental value from within the range of remaining possible values:

$$
\begin{aligned}
& \text { Perceived environment value } \\
& =\text { random value of (true environment value } \pm 180 \\
& \quad \times(1-\text { plasticity-habitat-sensitivity }))
\end{aligned}
$$

The multiplication with \pm 180 is done to potentially cover the full $360^{\circ}$ in a symmetric fashion around the true value. If plasticity-habitat-sensitivity is high (close to 1), the perceived value will closely approximate the true value of the environment, but stochasticity increases as plasticity-habitatsensitivity is lower (fig. 3). After assessing the local environment this way, the individuals develop a phenotype (starting from their genotype value) as close as possible to the perceived environment according to their plasticity-potential, hence minimizing the mismatch with the environment. The possible phenotypic change equals plasticity-potential $\times 180$ in the correct direction, so a plasticity-potential of 1 allows any mismatch to be fully corrected, while a very small value for plasticitypotential will allow only a small correction even if the individual has a high plasticity-habitat-sensitivity (fig. 3).

For dispersal, individuals explore a set of randomly selected habitat patches according to their dispersal-potential (the number of patches explored $=$ dispersal-potential $\times$ 100). Similar to plasticity, individuals then use their dispersal- 


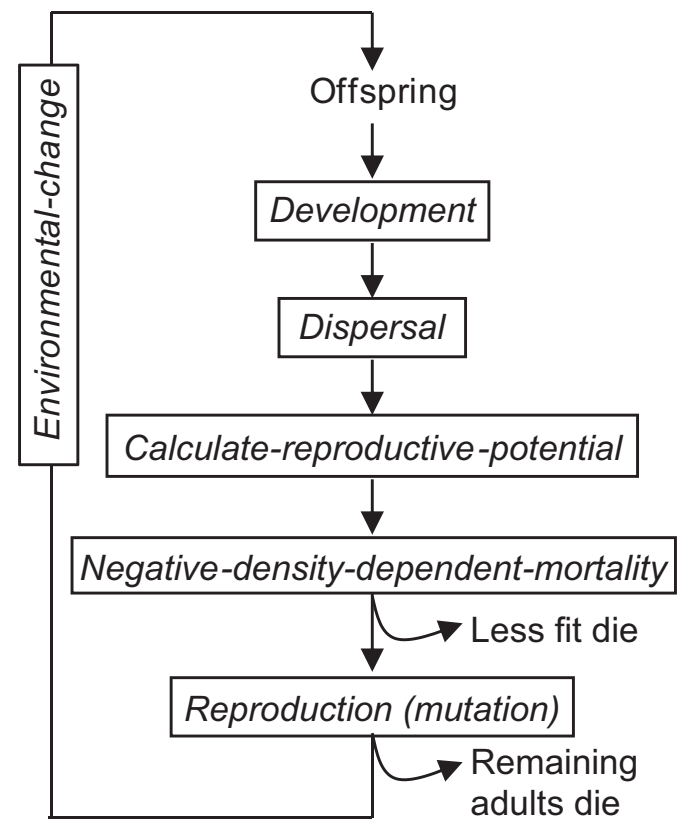

Figure 2: Overview of the main events across one generation of the model. Young individuals first develop and then disperse. After this, their reproductive potential is calculated according to their mismatch with the local environment and incurred trait costs. Next, if the local density is higher than carrying capacity, the excess individuals with the lowest reproductive potential die. Finally, individuals reproduce (with mutation) relative to their reproductive potential. After that, all adult individuals die, all local environments change, and the process starts again with the newborn offspring.

habitat-sensitivity to estimate the environment of these patches and their natal patch. With increasing dispersal-habitatsensitivity, estimated values get closer to the true environment. Then, individuals disperse to the patch with the lowest perceived mismatch between their phenotype and the environment, which might also be the natal patch (staying is also a dispersal decision). When dispersal-habitat-sensitivity is very low, dispersal is basically random with respect to the phenotype (see Edelaar and Bolnick 2012) and therefore does not result in MHC: for MHC, both sufficient dispersalpotential and sufficient dispersal-habitat-sensitivity are needed. Hence, MHC is modeled by two distinct traits, making the process as comparable as possible to plasticity as modeled here.

Phenotypic plasticity and MHC increase performance imperfectly if plasticity-potential or dispersal-potential are insufficient to bridge the gap between genotype and environment or if the environment is not perfectly perceived by the sensitivity traits. Plasticity and MHC can even be maladaptive if the individual makes an incorrect assessment of the environment, which is more likely with lower values of plasticity-habitat-sensitivity and dispersal-habitat-sensitivity (fig. 3).

\section{Selective Population Density Regulation}

When population size in a given habitat patch is above carrying capacity (>100 individuals), only the 100 individuals with the highest reproductive-potential (a parameter for expected fitness) survive until reproduction (fig. 1; see below). This negative density dependence imposes a strong selection on local performance, and since survival also depends on the performance of other individuals present (frequency dependence), together this increases the importance of natural selection in driving evolution in the model and reduces the importance of genetic drift.

\section{Selective Reproduction}

Modeling MHC and plasticity as a two-component process, whose elements may evolve independently, addresses the increasing recognition that different components of these processes may have different costs but that this is rarely taken into account (Auld et al. 2009; Travis et al. 2012; Duputié and Massol 2013; Delgado et al. 2014). Costs are important for the evolution and expression of dispersal and plasticity, so each of the two heritable traits linked to plasticity or MHC have an associated cost (a parameter imposed at model initialization for the entire simulation run and for all individuals). The modeled cost for dispersal-potential could be seen as the cost of development/maintenance of dispersal attributes but also as the cost of movement, as it influences the number of patches actually explored: even those individuals returning home pay these costs. The costs for the two plasticity traits can be viewed as costs for development and maintenance of the plasticity machinery (plasticity-potential) and those for information acquisition and processing (plasticityhabitat-sensitivity). These costs, which are equivalent in values and effects, together with the mismatch of the individual with the environment, reduce the reproductive-potential (the parameter for expected fitness) of individuals, which is calculated after dispersal and development as follows:

$$
\begin{aligned}
& \text { reproductive-potential } \\
& =1-(\mid \text { environment-phenotype } \mid / 180)-\mathrm{PP} \times \operatorname{cost}_{\mathrm{PP}} \\
& \quad-\mathrm{PHS} \times \operatorname{cost}_{\mathrm{PHS}}-\mathrm{DP} \times \operatorname{cost}_{\mathrm{DP}}-\mathrm{DHS} \times \operatorname{cost}_{\mathrm{DHS}},
\end{aligned}
$$

where PP stands for plasticity-potential, PHS for plasticityhabitat-sensitivity, DP for dispersal-potential, and DHS for dispersal-habitat-sensitivity and where the cost parameters can be specific for each trait. Reproductive-potential has a maximum of 1 and a minimum of 0 (any calculated negative values are nonsensical and rounded to 0 ). The costs increase linearly with the level of these traits, so, for example, 

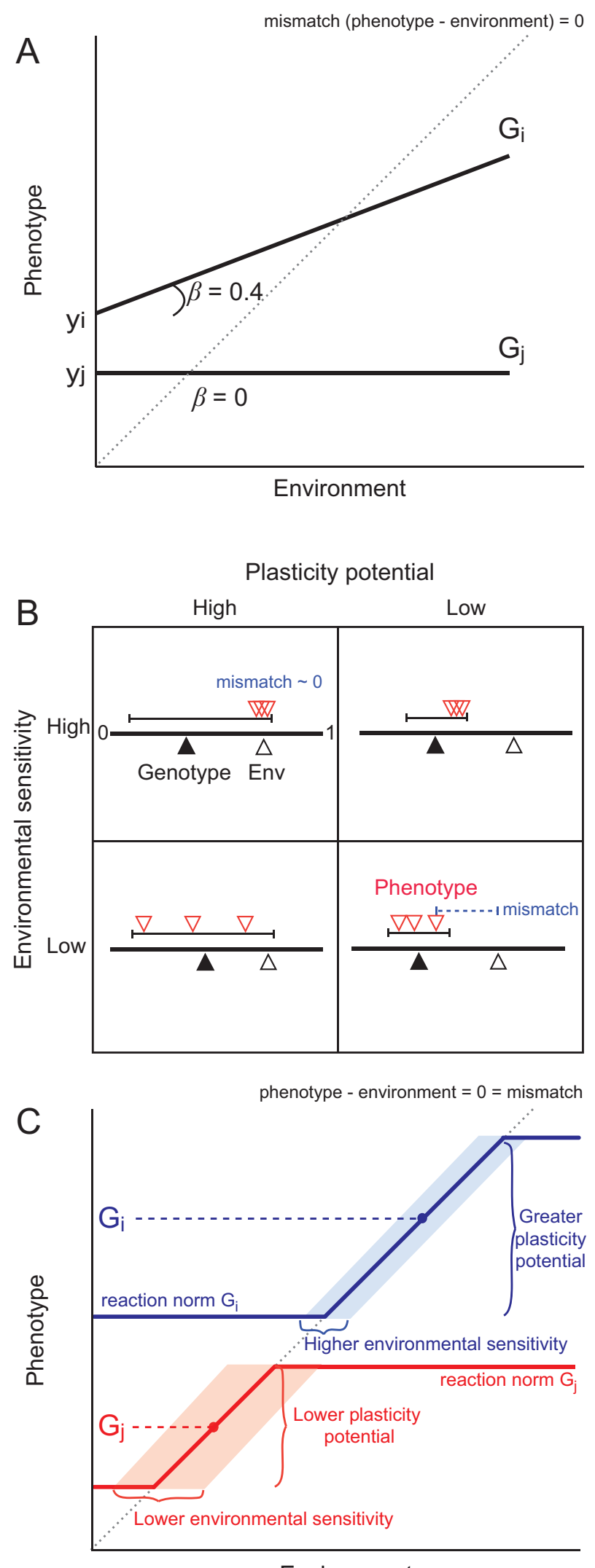

Environment if its cost parameter is nonzero, it is twice as costly to have a plasticity-potential of 0.70 versus 0.35 . The mismatch calculation uses the absolute difference between environment and phenotype, because the direction of the mismatch has the same fitness effect, and this difference is divided by 180 because this is the maximum difference in degrees possible for a circular trait: the mismatch therefore varies from 0 (perfectly adapted phenotype) to 1 (worst possible phenotype). This fitness function is a simple linear one, because here we do not wish to vary the strength of selection as a separate parameter. Reproductive-potential thereby varies from 0 to 1 , and the individuals with a higher reproductive-potential are those with a better match (smaller mismatch) to their environment, yet they suffer lower costs of trait production and maintenance associated with their capacity for MHC and plasticity. Such reduced costs could come from low trait values and/or reduced cost parameters.

Individuals that survived density regulation reproduce according to their reproductive-potential. Note that reproductive potential thus is selected upon twice: during densitydependent regulation and during reproduction. This further reduces the effect of genetic drift, provides a more realistic mixture of local and global selection as well as soft and hard selection (which can give different results; see Ravigné et al. 2009 and Débarre and Gandon 2011), and avoids the assumption that density regulation would be neutral with respect to performance. Individuals get the opportunity to produce two offspring, but each potential offspring is born if a randomly drawn number between 0 and 1 is lower than the reproductive-potential of the parent. Offspring inherit the five

Figure 3: $A$, Theoretical models of phenotypic plasticity often use a reaction norm approach where, for any given genotype $\left(G_{i, j}\right)$ plasticity is defined by the slope $\left(\beta_{i, j}\right)$ and intercept $\left(y_{i, j}\right)$ of the relationship between phenotype and environment. $B$, In our model, each genotype (filled triangle) has a plasticity potential that determines de range of phenotypes that it is capable of producing around its genotype (thin horizontal line). The true environmental state (Env) is given by the black open triangle (environment rescaled to run from 0 to 1 ; thick line). If environmental sensitivity is high (top left and top right), the organism will closely assess the true environment and will produce a phenotype (red open triangle) within its plasticity potential that highly reduces the mismatch (dotted line) with the estimated environment. However, as environmental sensitivity becomes lower (lower left and lower right), the organism will assess the environment with increasing inaccuracy, up to the point that it may take any random (and potentially maladaptive) phenotypic value allowed by its plasticity potential. $C$, An approximation of plasticity as modeled in this study using the reaction norm approach. Two individuals have an expected phenotype as determined by their respective genotypes $\mathrm{G}_{i}$ and $\mathrm{G}_{j}$. Within the limits set by the width of their individual plasticity potential, they will try to express a more adaptive phenotype (sloping parts of the reaction norm). Depending on habitat sensitivity (i.e., their ability to correctly assess the environmental state), there is more $\left(G_{j}\right)$ or less $\left(G_{i}\right)$ maladaptive noise in the produced phenotypes. If the estimated environment lies outside their plasticity potential, the most extreme phenotype possible is produced (horizontal parts of the reaction norm). 
traits from the parent with some mutation (see "Individual Characteristics"). After reproduction, all adults die, so generations do not overlap. Subsequently, the environment of each patch independently changes (see "Environment"), and the cycle starts again for the next generation.

\section{Simulations}

In a first set of simulations (results in figs. 4,5 ), we compared the ability of genetic differentiation, plasticity, and MHC to enhance local performance and allow population survival. Genetic differentiation is a default route to adap-

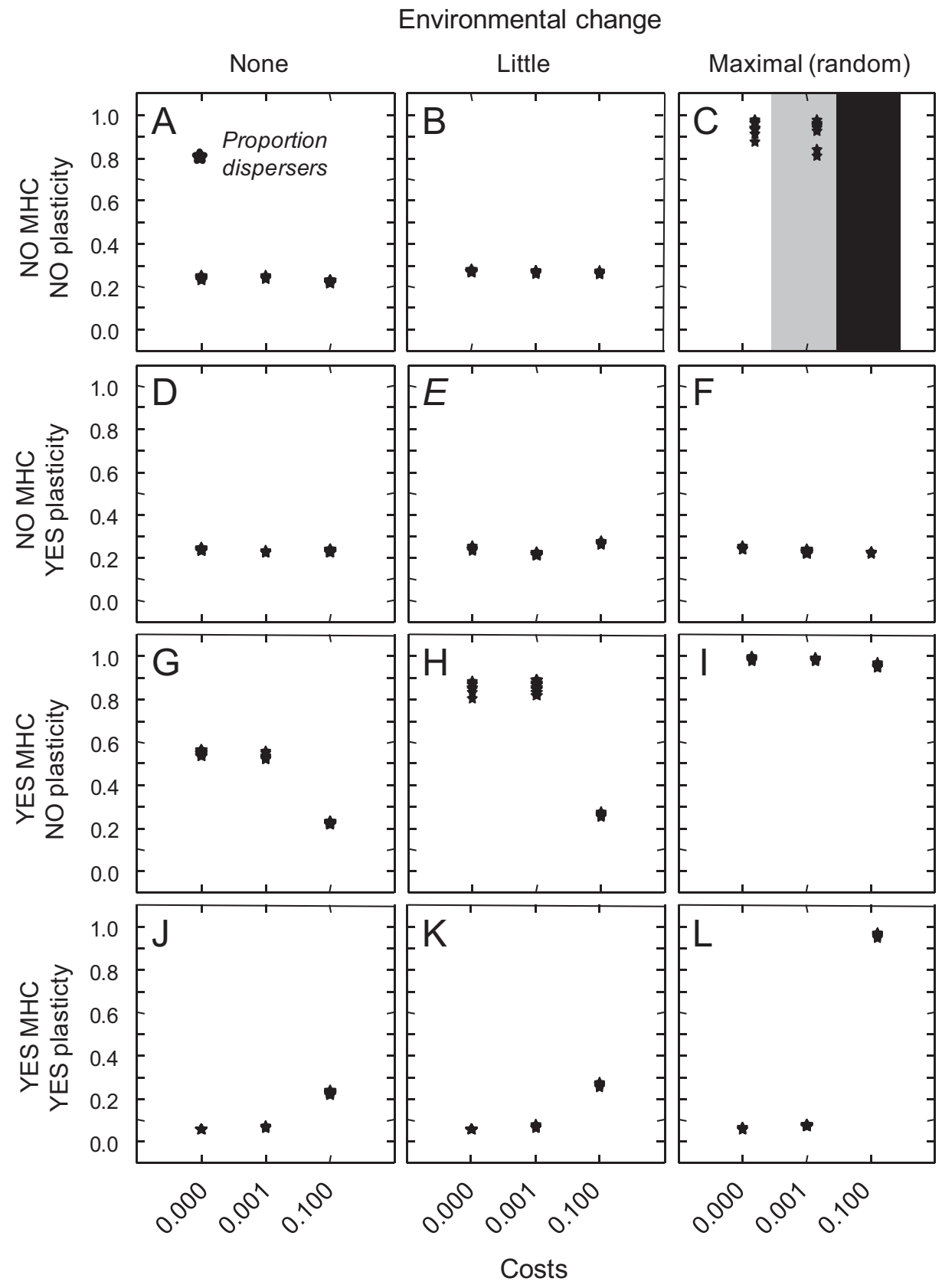

Figure 4: Population-level consequences of allowing plasticity and matching habitat choice (MHC) to evolve or not (rows), depending on the amount of environmental change (columns) and the costs for all traits underlying the modeled adaptations ( $X$-axis). Organisms develop first and then disperse. On the $Y$-axis, black stars are the proportion of individuals that dispersed away from the natal patch at the end of each of 10 independent runs. The colored background indicates when the entire metapopulation went extinct in part of the runs (gray) or all of the runs (black). 


\section{Environmental change}

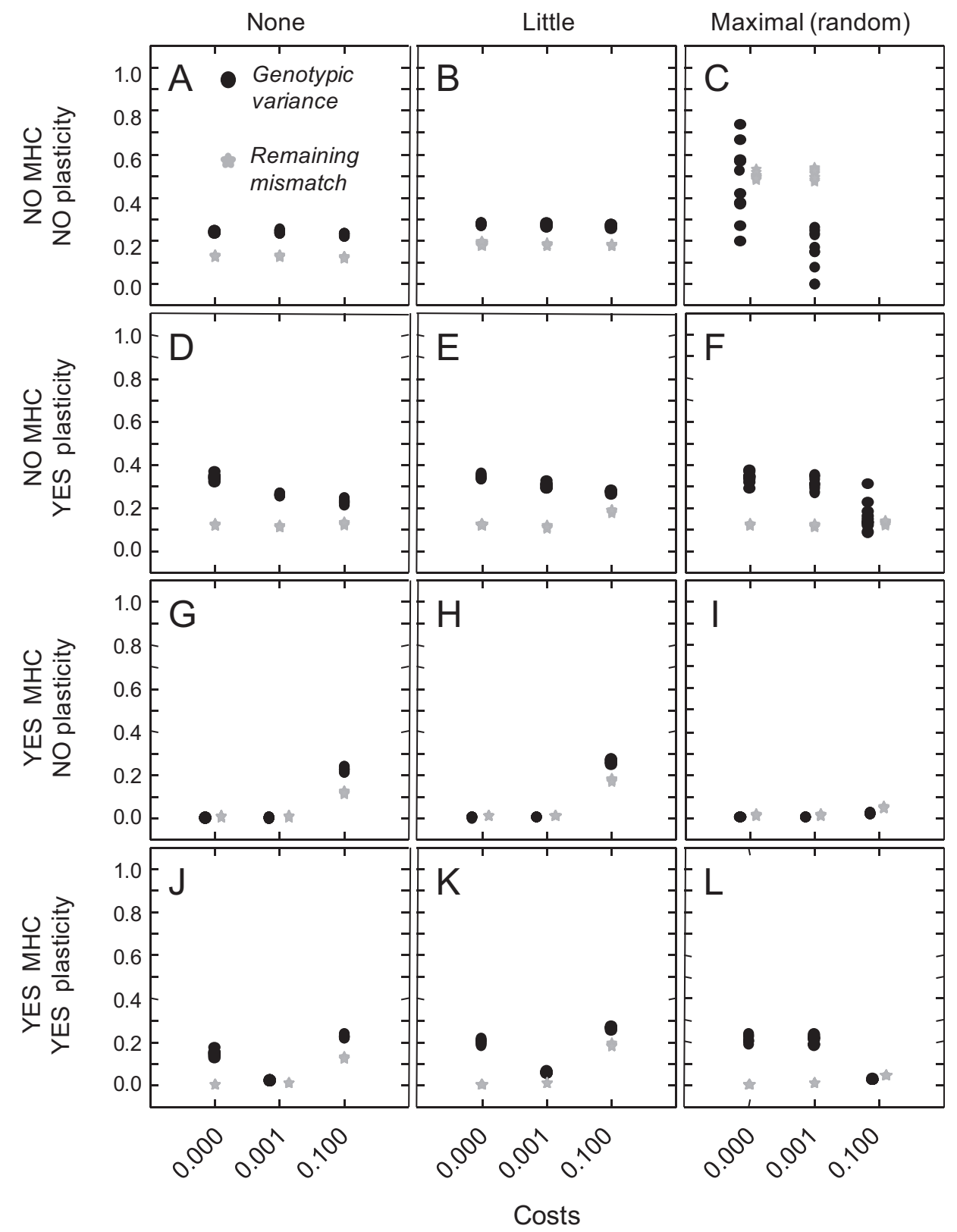

Figure 5: Population-level consequences of allowing plasticity and matching habitat choice to evolve or not (rows), depending on the amount of environmental change (columns) and the costs for all traits underlying the modeled adaptations ( $X$-axis). Black dots represent the within-patch genotypic variance, and gray stars are the remaining mismatch after plasticity and dispersal.

tation, which can always operate when heritable phenotypic variation is exposed to natural selection, as is the case here. To this default route, we added the possibility of evolving plasticity, evolving MHC, or evolving both simultaneously. As a kind of stress test, all three mechanisms of adaptation had to deal with increasing degrees of temporal environmental change (temporal-heterogeneity $=0$ [no change],
10 [little change], or change was fully random) and with the same increasing costs for all their underlying traits $(0,0.001$, or 0.1 ). As a measure of the degree of adaptation and persistence at the population level, we assessed the proportion of patches occupied and the degree of mismatch between phenotype and environment. To gain insight into the details of adaptation, we also assessed the proportion of individuals 
that dispersed (dispersal potential was always allowed to evolve) and the average genotypic variance per patch. Since the genotypes are circular values, we calculated circular genotypic variance as follows:

$$
1-\left\{\sqrt{ }\left[\left(\sum \cos g\right)^{2}+\left(\sum \sin g\right)^{2}\right] / N\right\}
$$

where $g$ are the genotypic values of all individuals, and $N$ is the number of individuals. This genotypic variance has a convenient range from 0 (all individuals identical) to 1 (all individuals regularly distributed along the circle).

In a second set of simulations (results in figs. 6, A1), we zoom in to the evolution of plasticity and MHC when both can evolve (i.e., how they interact with each other). We investigated whether the outcome depends on the cost of a focal plasticity or MHC trait $(0,0.001,0.1)$ and whether it depends on the degree of environmental change (relatively low with temporal-heterogeneity $=10$ vs. change being fully random). To evaluate the outcome, we extracted, for each simulation, the value of the four heritable traits of each individual alive after selection at the end of the run. For these simulations, the costs of the other three plasticity and MHC traits was held constant at a low value (0.001), which appears more realistic than no cost and avoids neutral evolution. The parameter for mutation (mean-mutational-change) was fixed at a moderate value of 0.01 .

Each simulation ran for 1,000 generations, which was confirmed to be enough for trait distributions to stabilize. Ten independent simulations were run per scenario, modeled to further investigate stability of the outcomes.

\section{Results}

Figures 4 and 5 confirm that adaptive plasticity and MHC can each greatly help populations to adapt to environmental heterogeneity. These two mechanisms of adaptation were superior to adaptation by genetic differentiation alone, which showed generally higher levels of mismatch (fig. 5; compare gray dots in fig. $5 A-5 C$ with those in $5 D-5 L$ ) and even extinction in some cases (fig. 4C). Plasticity and MHC thereby clearly represent viable solutions to the problem of increasing performance under environmental heterogeneity. However, they solve the problem in very different ways and with very different consequences. With plasticity only, dispersal evolved to low values (fig. 4), and since natural selection is relaxed by adaptively changing the phenotype, genetic variation within a population was maintained at a relatively high level (fig. 5). In contrast, with MHC only, dispersal evolved to high values (fig. 4), and each genotype settled in the population with the best-matching environment, so that individuals with similar genotypes chose similar patches, and genetic variation within a population was consequently low (fig. 5).
The costs of the underlying traits were clearly important. For adaptation by genetic differentiation only, the cost of dispersal could cause extinction (fig. 4C). For plasticity and $\mathrm{MHC}$, when the environments did not change or changed only a little, high costs to their two underlying traits (potential and sensitivity) precluded their evolution, and adaptation was instead solved by differential survival of nonplastic genotypes (see the similarity in results in figs. 4 and 5 when cost $=0.1$, and environmental change is "none" or "little"). This is because plasticity and MHC both require and therefore pay the cost of two traits, whereas dispersal potential was the only costly trait of relevance to adaptation by differential survival of nonplastic individuals.

When both plasticity and MHC were allowed to evolve, we again saw that, with high costs and little environmental change, adaptation occurred by genetic differentiation (figs. $4 \mathrm{~J}$, $4 K, 5 J, 5 K)$. However, for other combinations, the results were not similar to those of plasticity and $\mathrm{MHC}$ alone. For example, the dispersal rate was lower than for plasticity or MHC only (fig. $4 J-4 L$ ), and the pattern for genetic variance was also different (fig. 5J-5L). This confirms that, when these two mechanisms of adaptation can interact with each other, the outcomes are not easily deducible from models of each mechanism separately, and a joint model allowing for such more complex eco-evolutionary dynamics is necessary. The outcomes of these dynamics are explored in more depth in figures 6 and $\mathrm{A} 1$.

We explored how the degree of temporal environmental change and the costs for a specific focal trait affected the outcome. We first review the case when the degree of environmental change was low. As expected, at the highest cost level, all four traits evolved to very low values (fig. 6). Interestingly, however, the cost for one trait also influenced the evolution of the other traits. This was especially pronounced for the costs of the plasticity traits. When plasticity potential or plasticity habitat sensitivity had a high cost, then (and only then) dispersal potential flipped from being almost zero to almost one and, together with a high value for dispersal habitat sensitivity, combined to form a well-functioning MHC (fig. 6E, 6G). Another notable result was that plasticity potential increased when any of the MHC traits evolved to low values due to a high cost (fig. 6A, 6C), suggesting that, in that case, plasticity potential compensated for the disappearance of a fairly poor yet functional level of MHC. When still present, this MHC would act to increase local performance in the rare case that the environment changed so much that plasticity potential was too low to phenotypically track the change. A third striking aspect is that the sensitivity traits generally had high values (except when they themselves or their associated potential trait had a high cost).

When environmental change was maximal, the pattern for the MHC traits was virtually unchanged (fig. 6). The pat- 


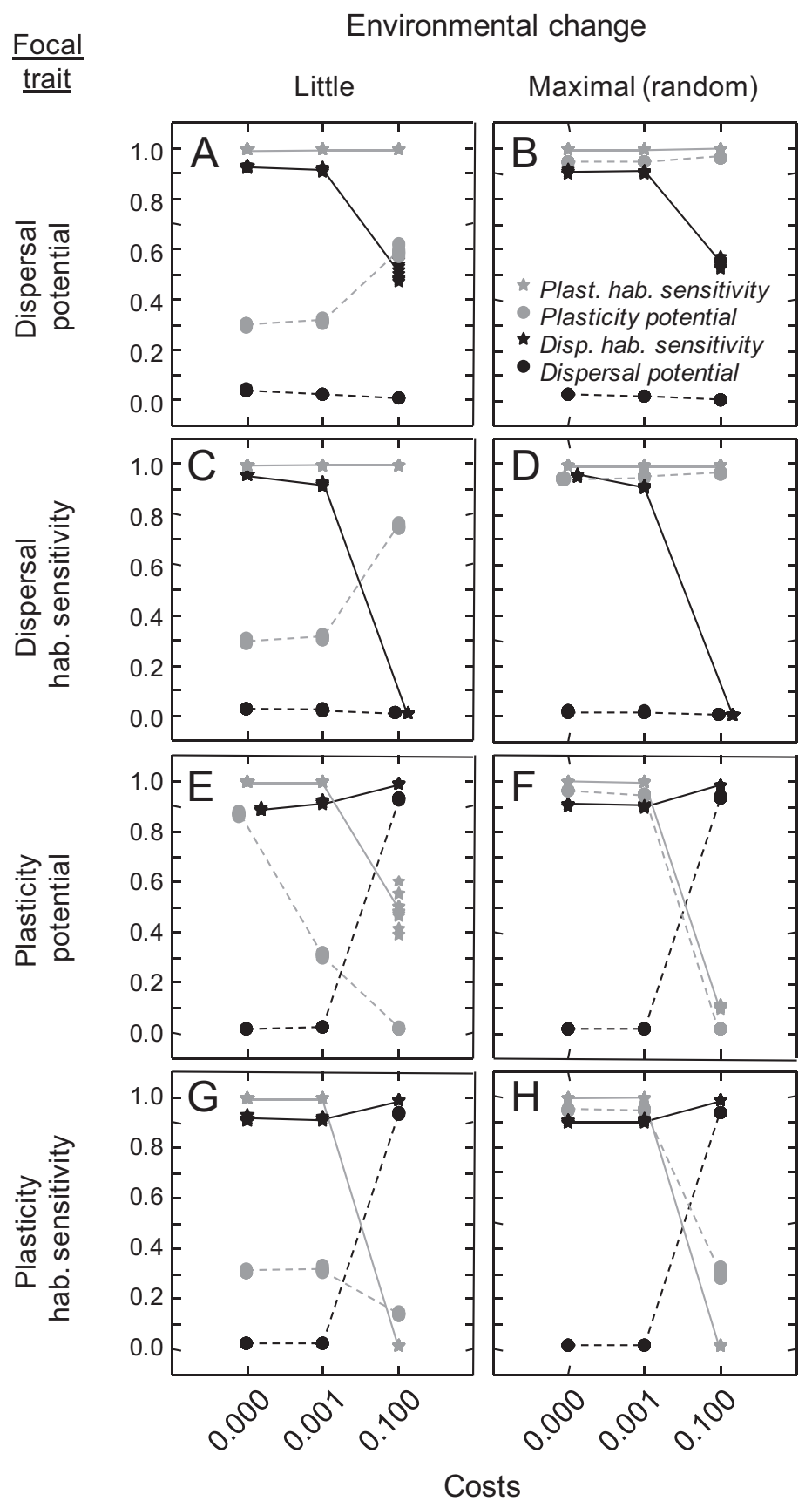

Figure 6: Values of the four underlying traits when development happens before dispersal and when both plasticity and matching habitat choice are allowed to evolve. We varied the degree of environmental change (little: $A, C, E, G$; maximal [random]: $B, D, F, H$ ). We also varied the cost of one trait (focal trait), whereas the other three traits have a constant low cost of 0.001 . Gray stars are plasticity habitat sensitivity, gray dots are plasticity potential, black stars are dispersal habitat sensitivity, and black dots are dispersal potential (averages for each of 10 runs).

tern was also very similar for plasticity habitat sensitivity. The biggest change was seen in the levels of plasticity potential that evolved: while the general patterns were similar, the absolute values were higher (fig. $6 F$ ) than with little environmental change, indicating that a greater local environmen- tal change implied that a broader plastic adjustment in the phenotype was required.

In summary, plasticity was the main mechanism allowing local performance to increase under selection. MHC provided a very minor but noticeable additional "safety net" in 
some situations. MHC became the prominent driver of local performance only when any one of the plasticity traits had a high cost and the evolution of plasticity was disfavored. The degree of environmental change had relatively little effect.

\section{Discussion}

Here we tested when and how metapopulations exposed to temporal variation evolve genetic differentiation due to natural selection, adaptive phenotypic plasticity, and/or a particular type of performance-increasing habitat choice: matching habitat choice (MHC). Based on the results of our model, which solutions evolve to increase local performance depends (i) much on the costs of the underlying traits, (ii) less on the degree of temporal environmental change, and (iii) relatively little on the ontogenetic sequence of development and dispersal. For the latter result, see figure A1; because both plasticity and MHC are independently capable of improving the phenotype-environment match, the order in which they appear did not seem to be very relevant. Nevertheless, certain interactions between these factors are present.

\section{Single Solutions Are Common}

In most simulated scenarios, a single main mechanism of adaptation was favored (differential survival of nonplastic and nonchoosing variants, plasticity, or MHC). Plasticity and MHC are additional, and often dominant, individual-level solutions that act on top of (and can eliminate the need for) the background population-level process of genetic differentiation through differential survival. Natural selection will always favor the evolution of individual solutions to mismatched phenotypes (maladaptation) as long as there are no constraints on such individual capacity and the benefits outweigh the costs.

We find that plasticity is more often the dominant mechanism of adaptation, compared with MHC (fig. 6). This was not easily predicted beforehand. The main reason appears to be that, with plasticity, the mismatch between a given genotype and its local environment can, in principle, be solved exactly by developing the required phenotype, provided that it is within the plasticity potential. Conversely, MHC can allow for a perfect match only if there is, in fact, a patch available that exactly corresponds to the phenotype of the individual. Therefore, some degree of phenotype-environment mismatch is more likely to remain under MHC than with plasticity. This appears to be a difference that will be relevant independently of specific model assumptions and real biological scenarios. Scheiner (2016) has also recently investigated the interaction between plasticity and habitat choice using a different modeling approach and also found that plasticity tends to evolve more commonly, likely in part because of this inherent advantage. However, the degree of remaining mismatch should depend on the environmental variability. In nonpatchy environments with smooth, predictable environmental gradients, MHC should be easier to effectuate for organisms and more effective as it can obtain a perfect phenotypeenvironment match, and it indeed has been found that it can produce and maintain steep clines (Armsworth and Roughgarden 2008). Similarly, in highly discrete situations with only two or a few types of environments, MHC can lead to the evolution of specialist genotypes with a high capacity of $\mathrm{MHC}$ and thereby of genetically differentiated populations, if not species (Bolnick and Otto 2013). Therefore, the extent of disadvantage of MHC with respect to plasticity may be highest at an intermediate number of environments available, as modeled here. Therefore, MHC might be more relevant and prevalent in nature when there are environmental gradients or when few discrete environments exist (e.g., Benkman 2017). Nonetheless, this hypothesis demands empirical studies and could also be tested with models that not only vary the number and spatial autocorrelation of patches but also allow for the evolution of MHC and plasticity at the same time and in an equivalent manner, which typically is not done in habitat choice models. Our model (model code and executable file available at http://dx.doi.org/10.5061/dryad.g370c) could be used as a basis for this expansion.

\section{Costs Can Have Important Limiting Effects}

Costs of the underlying traits heavily determine which of the three mechanisms of adaptation is favored and also that well-developed plasticity and MHC are never found together. When the traits underlying plasticity and MHC have a high cost and environmental variability is low or absent (i.e., there is little benefit), adaptation occurs via genetic differentiation only (figs. $4,5 E, 5 H$ ). This makes good sense and should be independent of specific model assumptions. When environmental change is greater, costly traits that do not yield sufficient additional benefits are selected against, regardless of the order in which they occur during their life cycle. Thus, knowing in more detail to what extent MHC and plasticity can provide sufficient phenotype-environment match is crucial: if the solutions remain imperfect, then additional gain might be achieved via the evolution of a mixed (sequential) strategy (e.g., fig. 6A, 6C).

Other authors have also pointed out the importance of the underlying trait costs for the understanding of the evolution and expression of dispersal or plasticity (e.g., DeWitt et al. 1998; Stamps et al. 2005; Bonte et al. 2012). There is comparatively good knowledge on how variation in the environment affects functional adaptation (e.g., biomechanical models or selection gradients), but there is a relatively poor understanding of the costs of traits. We know of no biological system in which good estimates of both plasticity and dispersal costs have been measured. Nonetheless, dispersal costs have been well documented and seem to be common, multifari- 
ous, and often substantial (reviewed in Bonte et al. 2012). On the other hand, substantial costs of plasticity have been more elusive to document, and costs are instead often absent or even of opposite sign (reviewed in Murren et al. 2015). (Note, however, that there might be bias against detecting high costs, because our results show that high costs select for the absence of the mechanism of which they are a component: plasticity costs may be high in systems without plasticity.) This suggests that perhaps the empirical costs for MHC are generally higher than for plasticity. If so, this may further explain why adaptive plasticity may be more common than MHC. However, the scarcity of focused studies and the fact that MHC is harder to detect than plasticity could also partly bias our perception of its occurrence in nature.

It is increasingly stressed that it is important to recognize that separate components of the dispersal and plasticity process may have different costs, as we have modeled here (Auld et al. 2009; Travis et al. 2012; Duputié and Massol 2013; Delgado et al. 2014). On doing so, we have found that high costs for any of the involved traits of a composite mechanism make its evolution less likely, showing that isolated costs for single components can have large spillover effects onto apparently unrelated traits. This is clear if we understand organisms and not traits as the targets of selection (e.g., Phillips and Arnold 1989), but it is easily (and still often) overlooked when studying traits in isolation in nature or in theoretical studies.

\section{Mechanisms of Adaptation Influence Genetic Variation and Population Structuring}

Since phenotypic plasticity enables adaptive phenotypes to be expressed beyond the fixed effect of the genotype and thereby shields them from natural selection, it helps in preserving genetic variation within populations (Gomez-Mestre and Jovani 2013; fig. 5). At the same time, it contributes to preventing genetic differentiation among populations (Thibert-Plante and Hendry 2010). In contrast, MHC has the ability to cluster similar genotypes into local habitats, which reduces genetic variation within populations (fig. 5) but increases genetic differentiation among populations (Bolnick and Otto 2013). However, since the increased genotype-environment match provided by MHC also acts to shield genotypes from natural selection, genetic variation at the metapopulation level is maintained, and the evolution of a generalist genotype that has highest fitness across environments is avoided. Both these mechanisms may therefore contribute to the maintenance of genetic variation, a topic of great historical and applied interest (Hedrick 2006).

\section{Robustness of Results}

Models of dispersal have typically assumed a fixed rate of dispersal and that individuals have either random move- ment or perfect knowledge of the environment and optimal dispersal (Bowler and Benton 2005; Benton and Bowler 2012; Delgado et al. 2014). Moreover, dispersal models generally do not let the morphological or behavioral characteristics of organisms evolve, which would consequently cause movement rules to evolve (Starrfelt and Kokko 2012; Delgado et al. 2014; Berner and Thibert-Plante 2015). The model we present here avoids these limitations. Moreover, we modeled local population sizes of up to 100 individuals in each of 100 patches exchanging individuals, which should reduce genetic drift and increase the response to selection. Indeed, given the great similarity in outcome among the 10 independent simulation runs with the same parameter settings (see figs. 4-6), we conclude that drift has not affected the general patterns we report here.

To our knowledge, the study by Scheiner (2016) is the only other study modeling the interaction among evolving plasticity, MHC and genetic differentiation, but with important differences. First, we focus on the simultaneous evolution and operation of comparable but distinct mechanisms of adaptation, whereas Scheiner (2016) focuses on how the presence of habitat choice affects the evolution of plasticity. That study also categorizes outcomes on the basis of the genetics of fixed and plastic effects on the phenotypes (i.e., how much genetic differentiation and plasticity is there). For example, population differentiation in fixed genetic effects can be caused by divergent natural selection and the absence of dispersal or by MHC involving much dispersal. In Scheiner (2016), these different routes are classified together on the basis of their identical outcomes, whereas we clearly differentiate them on the basis of their different underlying mechanisms. We feel that, when assessing empirical systems, it is important to make this distinction between cause and effect and to realize that the same pattern may have different causes. Second, some of the modeling choices made in Scheiner (2016) also differ from our own. For example, we modeled costs and error identically for habitat choice and plasticity. Importantly, individuals compare habitats simultaneously and thereby can move directionally toward those with better matching instead of comparing random habitats sequentially: maximally choosing individuals are therefore much more likely to leave their natal habitat and find a better matching habitat, thereby equalizing the playing field for plasticity and habitat choice. In addition, in our model, environments and genotypes are not ordered linearly but in a circular fashion (i.e., they are not bounded). This excludes the evolution of a "jack-of-all-trades," which is a single generalist genotype that, on average, performs best across all environments (i.e., a genotype that is not locally adapted to the current environment but adapted to a fixed range of environments that it will encounter across time). However, in essence, this difference is minor, because both in Scheiner (2016) and in our study, local adaptation by genetic differentiation via natural 
selection is possible (and observed), and plasticity and habitat choice may or may not evolve.

Despite the differences in approach, several important results are consistent across both studies: (i) high temporal variation without plasticity or habitat choice leads to population extinction, and higher temporal variation favors the evolution of plasticity or habitat choice; (ii) when there is more temporal variation, there is more dispersal; (iii) with a higher dispersal cost, there is less random dispersal; (iv) if plasticity occurs after the environmental change, then plasticity is the favored solution to environmental change; and (v) the reliability of environmental assessment is important to the evolution of habitat choice, just as for plasticity.

The first three similarities could be seen as validations of the realism of both models. That plasticity is such a dominant outcome, however, is less intuitive. As discussed above, this may be because MHC is more likely to result in a remaining mismatch due to the discrete nature of available environments.

The importance of the reliability of environmental cues is often mentioned as important for the evolution of plasticity (e.g., Tufto 2000; Reed et al. 2010). Our observations add to this that it is not only the reliability of the cues given by the environments that matters but also the reliability of the perception of these cues. Counterintuitively, high perception ability may evolve to prevent plasticity or MHC from operating when the match is already very good. The encountered importance of assessment reliability also leads to the hypothesis that, in nature, it may be relatively harder to obtain accurate assessments for multiple patches while (briefly) exploring for MHC than for the single (resident) home patch for plasticity. We have not modeled this possibility, but if so, in real systems, this constraint/greater cost may further reduce the probability that MHC evolves instead of plasticity.

To what extent the results are influenced by kin-competition has not been quantified in either study. Kin competition can be a major driver for the evolution of dispersal (Hamilton and May 1977) and is able to operate in our model. MHC tends to bring similar genotypes (including relatives) together again in the same habitat patches, even if they all left the natal patch, which might increase kin competition after dispersal. However, the great number of patches and of individuals per patch as modeled here will reduce kin competition. It should also be realized that less temporal variation and the evolution of plasticity favor residency, which also increases kin competition. On balance, the exact relevance of kin competition to these and other results is therefore unknown but is likely to be small, although it might be interesting to investigate to what extent kin competition may act as additional costs in the evolution of plasticity and habitat choice and under which conditions.

The type of habitat choice modeled here and in Scheiner (2016) is MHC. Other types of habitat choice that could im- prove local performance in relation to the phenotype are habitat choice due to specific preference alleles or due to familiarity or imprinting. Berner and Thibert-Plante (2015) highlight that, when investigated in isolation, the specific mechanism underlying habitat choice can have large effects on its likelihood to evolve and on its effect to promote adaptive population genetic divergence. These different types of habitat choice may therefore also have different interactions with plasticity. A next step to further model the simultaneous evolution of multiple solutions to single problems could therefore be to investigate how each type of habitat choice interacts with plasticity and how they all interact with each other.

\section{Conclusions}

Based on our results and those of Scheiner (2016), we now have a general idea of when to expect genetic differentiation, plasticity, or MHC to be responsible for increasing the fit between individual phenotypes and changing environmental conditions in nature. Genetic differentiation via natural selection on nonplastic phenotypes is the default route to phenotype-environment match when environmental fluctuations are small and costs of plasticity and dispersal are considerable. However, as environmental heterogeneity increases, plasticity and MHC are favored as long as their costs are sufficiently small. However, of these two, plasticity is the default evolving mechanism, especially when mismatch remains after MHC. The evolution of plasticity may be prevented (i) when there are high costs for any of the traits required for plasticity, (ii) when the environment changes after development has already finished, or (iii) when rates of environmental change are greater than the ability to plastically alter the phenotype. In those cases, MHC may evolve as an alternative or additive mechanism for increasing local performance. The evolution of MHC is in turn problematic (i) when the costs for the underlying traits (including dispersal itself) are too high, (ii) when it is hard to obtain accurate information on performance in the environments available (e.g., environments far away, visited briefly, or involving functional traits whose effect on local performance is hard to perceive), (iii) when dispersal occurs before environmental change, (iv) when adaptation is sought for several traits at the same time (an optimal habitat for several traits less likely to be found or even exist), or (v) when MHC is otherwise constrained, such as when environments change quicker than can be tracked (either for information collection or for actual movement) or when strong territoriality prevents free movement. This long list of inhibiting factors for MHC may explain its apparent rarity in nature relative to plasticity. But at the same time, it can be used to identify empirical systems in which plasticity is less likely and MHC should be 
expected, or at least should not be excluded a priori, as is often done.

\section{Acknowledgments}

We thank all anonymous reviewers for comments; Sam Scheiner for comments and access to his unpublished manuscript; and Marion Nicolaus, Pierre-André Crochet, LuisMiguel Chevin, Ophélie Ronce, and Ulf Dieckmann for feedback. This work was supported by the Spanish Ministry of Economy and Competitiveness (RYC-2011-07889, CGL2012-35232, CGL2013-49460-EXP, and CGL2016-79483-P to P.E.; CGL2014-59206-P to I.G.-M.; RYC-2009-03967 to R.J.) with support from the European Regional Development Fund.

\section{Literature Cited}

Armsworth, P. R., and J. E. Roughgarden. 2008. The structure of clines with fitness-dependent dispersal. American Naturalist 172: 648-657.

Auld, J. R., A. A. Agrawal, and R. A. Relyea. 2009. Re-evaluating the costs and limits of adaptive phenotypic plasticity. Proceedings of the Roval Society B 277:503-511.

Benkman, C. W. 2017. Matching habitat choice in nomadic crossbills appears most pronounced when food is most limiting. Evolution 71 (3):778-785. doi:10.1111/evo.13146.

Benton, T. G., and D. E. Bowler. 2012. Dispersal in invertebrates: influences on individual decisions. Pages 41-49 in J. Clobert, M. Baguette, T. G. Benton, and J. M. Bullock, eds. Dispersal ecology and evolution. Oxford University Press, Oxford.

Berner, D., and X. Thibert-Plante. 2015. How mechanisms of habitat preference evolve and promote divergence with gene flow. Iournal of Evolutionary Biology 28:1641-1655.

Bolnick, D. I., and S. P. Otto. 2013. The magnitude of local adaptation under genotype-dependent dispersal. Evolutionary Ecology 3:47224735.

Bonte, D., H. Van Dyck, J. M. Bullock, A. Coulon, M. Delgado, M. Gibbs, V. Lehouck, et al. 2012. Costs of dispersal. Biological Reviews 87:290-312.

Bowler, D. E., and T. G Benton. 2005. Causes and consequences of animal dispersal strategies: relating individual behavior to spatial dynamics. Biological Reviews 80:205-225.

Débarre, F., and S. Gandon. 2011. Evolution in heterogeneous environments: between soft and hard selection. American Naturalist 177:E84-E97.

Delgado, M. M., K. A. Barton, D. Bonte, and J. M. J. Travis. 2014 Prospecting and dispersal: their eco-evolutionary dynamics and implications for population patterns. Proceedings of the Roval Societv B 281:20132851.

DeWitt, T. J., A. Sih, and D. S. Wilson. 1998. Costs and limits of phenotypic plasticity. Trends in Ecology and Evolution 13:77-81.

Duputié, A., and F. Massol. 2013. An empiricist's guide to theoretical predictions on the evolution of dispersal. Interface Focus 3:20130028.

Edelaar, P., and D. I. Bolnick. 2012. Non-random gene flow: an underappreciated force in ecology and evolution. Trends in Ecology and Evolution 27:659-665.
Edelaar, P., R. Jovani, and I. Gomez-Mestre. 2017. Data from: Should I change or should I go? phenotypic plasticity and matching habitat choice in the adaptation to environmental heterogeneity. American Naturalist, Dryad Digital Repository, http://dx.doi.org/10.5061 /dryad.g370c.

Edelaar, P., A. M. Siepielski, and J. Clobert. 2008. Matching habitat choice causes directed gene flow: a neglected dimension in evolution and ecology. Evolution 62:2462-2472.

Eyre-Walker, A., and P. D. Keightley. 2007. The distribution of fitness effects of new mutations. Nature Reviews Genetics 8:610-618.

Gomez-Mestre, I., and D. R. Buchholz. 2006. Developmental plasticity mirrors differences among taxa in spadefoot toads linking plasticity and diversity. Proceedings of the National Academy of Sciences of the USA 103:19021-19026.

Gomez-Mestre, I., and R. Jovani. 2013. A heuristic model on the role of plasticity in adaptive evolution: plasticity increases adaptation, population viability and genetic variation. Proceedings of the Roval Societv B 280:20131869.

Hamilton, W. D., and R. M. May. 1977. Dispersal in stable habitats. Nature 269:578-581.

Hedrick, P. W. 2006. Genetic polymorphism in heterogeneous environments: the age of genomics. Annual Reviews in Ecology, Evolution and Systematics 37:67-93.

Hendry, A. P. 2015. Key questions on the role of phenotypic plasticity in eco-evolutionary dynamics. Journal of Heredity 107:25-41.

Kawecki, T. J., and D. Ebert. 2004. Conceptual issues in local adaptation. Ecology Letters 7:1225-1241.

Karpestam, E., L. Wennersten, and A. Forsman. 2012. Matching habitat choice by experimentally mismatched phenotypes. Evolutionary Ecology 26:893-907.

Moczek, A. P., S. Sultan, S. Foster, C. Ledon-Rettig, I. Dworkin, H. F. Nijhout, E. Abouheif, and D. W. Pfennig. 2011. The role of developmental plasticity in evolutionary innovation. Proceedings of the Roval Society B 278:2705-2713.

Morin, P. J. 2011. Habitat selection. Pages 238-250 in P. J. Morin, ed. Community ecology. 2nd ed. Wiley, Chichester.

Morris, D. W. 2003. Towards an ecological synthesis, a case for habitat selection. Oecologia 136:1-13.

Murren, C. J., J. R. Auld, H. Callahan, C. K. Ghalambor, C. A. Handelsman, M. A. Heskel, J. G. Kingsolver, et al. 2015. Constraints on the evolution of phenotypic plasticity: limits and costs of phenotype and plasticity. Heredity 115:293-301.

Phillips, P. C., and S. Arnold. 1989. Visualizing multivariate selection. Evolution 43:1209-1222.

Pigliucci, M. 2001. Phenotypic plasticity: beyond nature and nurture. Johns Hopkins University Press, Baltimore.

Räsänen, K., and A. Hendry. 2008. Disentangling interactions between adaptive divergence and gene flow when ecology drives diversification. Ecology Letters 11:624-636.

Ravigné, V., U. Dieckmann, and I. Olivieri. 2009. Live where you thrive: joint evolution of habitat choice and local adaptation facilitates specialization and promotes diversity. American Naturalist 174:E141E169.

Ravigné, V., I. Olivieri, and U. Dieckmann. 2004. Implications of habitat choice for protected polymorphisms. Evolutionary Ecology Research 6:125-145.

Reed, T. E., R. S. Waples, D. E. Schindler, J. J. Hard, and M. T. Kinnison. 2010. Phenotypic plasticity and population viability: the importance of environmental predictability. Proceedings of the Roval Societv B 277:3391-3400. 
Rose, M. R., and G. V. Lauder. 1996. Adaptation. Academic Press, Amsterdam.

Rueffler, C., T. J. M. Van Dooren, O. Leimar, and P. A. Abrams. 2006. Disruptive selection and then what? Trends in Ecology and Evolution 21:238-245.

Scheiner, S. M. 2013. The genetics of phenotypic plasticity. XII. Temporal and spatial heterogeneity. Ecologv and Evolution 3:4596-4609. - 2016. Habitat choice and temporal variation alter the balance between adaptation by genetic differentiation, a jack-of-all-trades strategy, and phenotypic plasticity. American Naturalist 187:633646.

Scheiner, S. M., M. Barfield, and R. D. Holt. 2012. The genetics of phenotypic plasticity. XI. Joint evolution of plasticity and dispersal rate. Ecology and Evolution 2:2027-2039.

Stamps, J. A., V. V. Krishnan, and M. L. Reid. 2005. Search costs and habitat selection by dispersers. Ecology 86:510-518.

Starrfelt, J., and H. Kokko. 2012. The theory of dispersal under multiple influences. Pages 19-28 in J. Clobert, M. Baguette, T. G. Benton, and J. M. Bullock, eds. Dispersal ecology and evolution. Oxford University Press, Oxford.

Sultan, S. E., and H. G. Spencer. 2002. Metapopulation structure favors plasticity over local adaptation. American Naturalist 160:271283.
Thibert-Plante, X., and A. P. Hendry. 2010. The consequences of phenotypic plasticity for ecological speciation. Iournal of Evolutionary Biology 24:326-342.

Tufto, J. 2000. The evolution of plasticity and nonplastic spatial and temporal adaptations in the presence of imperfect environmental cues. American Naturalist 156:121-130.

Travis, J. M. J., K. Mustin, K. A. Barton, T. G. Benton, J. Clobert, M. M. Delgado, C. Dytham, et al. 2012. Modeling dispersal: an eco-evolutionary framework incorporating emigration, movement, settlement behavior and the multiple costs involved. Methods in Ecology and Evolution 3:628-641.

West-Eberhard, M. J. 2003. Developmental plasticity and evolution. Oxford University Press, Oxford.

Wilensky, U. 1999. NetLogo. Center for Connected Learning and Computer-Based Modeling, Northwestern University, Evanston, IL. http://ccl.northwestern.edu/netlogo.

Wright, S. 1931. Evolution in Mendelian populations. Genetics 16:97159.

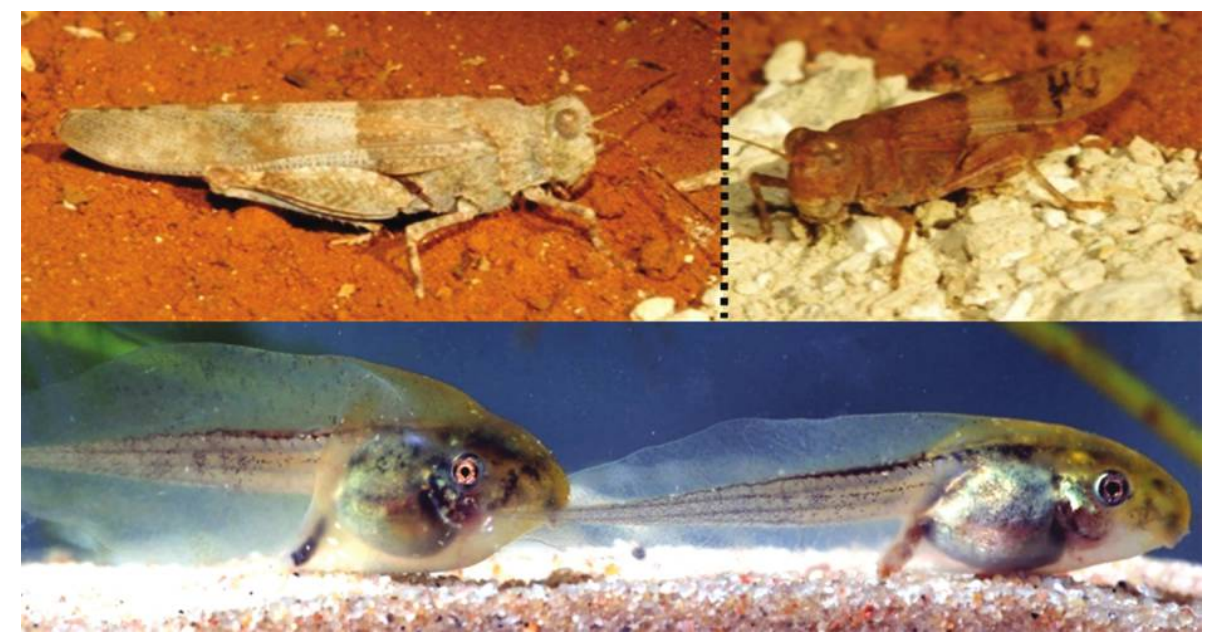

Top, two azure sand grasshoppers (Sphingonotus azurescens) and two distinct natural substrates: one substrate that matches a grasshopper's color and therefore provides protective crypsis, and another one that does not. Grasshoppers could increase crypsis by changing their color (adaptive phenotypic plasticity) or by moving to a suitable substrate (matching habitat choice). Photographs by Pim Edelaar. Bottom, two sibling tadpoles of the western spadefoot toad (Pelobates cultripes) with widely divergent phenotypes. The tadpole on the left was raised in the presence of chemical cues from a predatory dragonfly and developed a rounder body, a deeper tail fin, and a more anterior insertion of the tail than its sibling, which was raised in clean water (plasticity). Bottom photograph by Ivan Gomez-Mestre. 